\title{
Wireless Intraocular Pressure Sensing Using Microfabricated Minimally Invasive Flexible-Coiled LC Sensor Implant
}

\author{
Po-Jui Chen, Member, IEEE, Member, ASME, Saloomeh Saati, Rohit Varma, \\ Mark S. Humayun, Member, IEEE, and Yu-Chong Tai, Fellow, IEEE
}

\begin{abstract}
This paper presents an implant-based wireless pressure sensing paradigm for long-range continuous intraocular pressure (IOP) monitoring of glaucoma patients. An implantable parylene-based pressure sensor has been developed, featuring an electrical LC-tank resonant circuit for passive wireless sensing without power consumption on the implanted site. The sensor is microfabricated with the use of parylene $\mathrm{C}$ (poly-chlorop-xylylene) to create a flexible coil substrate that can be folded for smaller physical form factor so as to achieve minimally invasive implantation, while stretched back without damage for enhanced inductive sensor-reader coil coupling so as to achieve strong sensing signal. A data-processed external readout method has also been developed to support pressure measurements. By incorporating the LC sensor and the readout method, wireless pressure sensing with $1-\mathrm{mmHg}$ resolution in longer than $2-\mathrm{cm}$ distance is successfully demonstrated. Other than extensive on-bench characterization, device testing through six-month chronic in vivo and acute $e x$ vivo animal studies has verified the feasibility and efficacy of the sensor implant in the surgical aspect, including robust fixation and long-term biocompatibility in the intraocular environment. With meeting specifications of practical wireless pressure sensing and further reader development, this sensing methodology is promising for continuous, convenient, direct, and faithful IOP monitoring.

[2009-0111]
\end{abstract}

Index Terms-Implantable microdevice, intraocular pressure (IOP), LC pressure sensor, parylene, wireless sensing.

\section{INTRODUCTION}

$\mathbf{G}$ LAUCOMA is a debilitating eye disease that chronically damages the optic nerve and results in loss of vision for tens of millions of people worldwide [1]. The disease is associated with abnormally accumulated intraocular fluid

Manuscript received April 29, 2009; revised October 28, 2009; accepted April 21, 2010. Date of publication June 1, 2010; date of current version July 30, 2010. This work was supported in part by the Engineering Research Centers Program of the National Science Foundation (Award EEC-0310723) and in part by Bausch and Lomb. Subject Editor K. Najafi.

P.-J. Chen was with the Department of Electrical Engineering, Division of Engineering and Applied Science, California Institute of Technology, Pasadena, CA 91125 USA. He is now with the Research and Technology Center, Robert Bosch LLC, Palo Alto, CA 94304 USA (e-mail: pjchen@mems.caltech.edu).

S. Saati, R. Varma, and M. S. Humayun are with the Keck School of Medicine, University of Southern California, Los Angeles, CA 90033 USA, and also with the Doheny Eye Institute, Los Angeles, CA 90033 USA.

Y.-C. Tai is with the Department of Electrical Engineering and the Department of Bioengineering, Division of Engineering and Applied Science, California Institute of Technology, Pasadena, CA 91125 USA.

Color versions of one or more of the figures in this paper are available online at http://ieeexplore.ieee.org.

Digital Object Identifier 10.1109/JMEMS.2010.2049825 and resulting elevated intraocular pressure (IOP). Accordingly, successful IOP monitoring is crucial in the management of glaucoma patients as it is known to be one of the most effective methods to evaluate the progression of this eye disease. Current clinical diagnosis involves contact or noncontact applanation tonometry for IOP recording. However, both modalities have difficulties in providing reliable and repeatable measurements and, particularly, in deployment for regular (e.g., daily) tracking, which impedes prompt detection and appropriate treatment for IOP spikes from its diurnal fluctuations considered as a separate risk factor to optic nerve damage [2]-[4]. Continuous IOP monitoring in glaucoma patients with high accuracy and high reliability is therefore a consistent need for ophthalmologists.

Telemetric sensing is one of the viable methods to accomplish continuous and faithful noncontact IOP measurements [5], [6]. It utilizes a transensor implant that registers environmental pressure variations inside the eye so that the IOP can be directly measured by using an external reader wirelessly interrogating the implant. This methodology enables straightforward IOP sensing without involving further calculations which are derived from ocular mechanics as are used in applanation tonometry and which have large variation due to different dimensions and mechanical properties of individual eyes [7], [8]. In contrast to active sensing in which power transfer, size, and cost of the device are critical concerns [9], [10], passive sensing approaches have relatively flexible design constraints on the device side [11]. Works from many research groups have demonstrated the possibility of using miniaturized LC sensors (sensors with electrical LC resonant circuit) to enable passive wireless pressure sensing for various applications, including transcutaneous pressure monitoring, intracranial pressure monitoring, and pressure monitoring of abdominal aortic aneurysms in addition to the proposed IOP monitoring [12]-[17]. These devices serve as excellent examples, showing the potential of using such wireless passive pressure transensors for continuous measurement of physiological parameters in biomedical systems and human healthcare. Given the knowledge of wireless LC sensing from the literatures and that of exploiting parylene in integrated microsensors [18], we have developed a monolithically microfabricated parylene-based wireless passive pressure sensor in a form factor suitable for minimally invasive device implantation [19]. However, despite successful wireless pressure sensing demonstration, the sensing distance of the entire sensor-reader system in the previous work was not sufficient in 


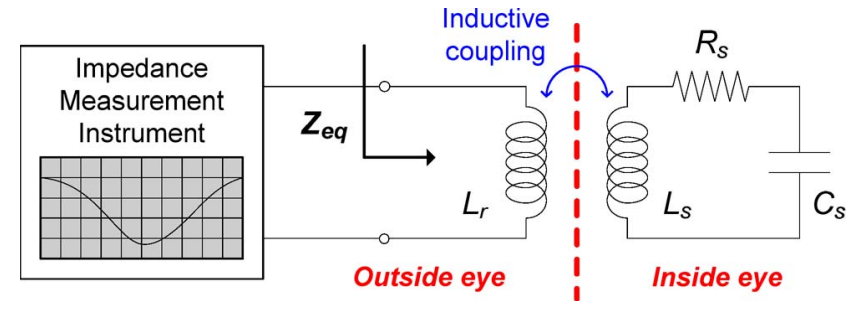

Fig. 1. Passive wireless IOP sensing concept. The implanted sensor can faithfully register pressure variations using corresponding electrical characteristic changes, which are measured using an external reader through a wireless inductive coupling link. (a)

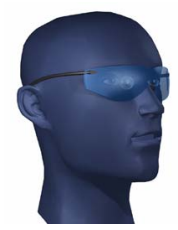

(b)

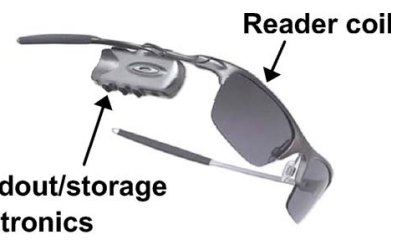

Fig. 2. Proposed IOP monitoring method in practice. (a) Autonomous and continuous sensing using wearable reader paradigm (e.g., glasses). (b) Integrated reader components on the glasses frame.

practice. Such challenge can also be found in other works with considering size and other constraints [6]. As a result of further improvement, this work focuses on a different sensor design for electromagnetic coil coupling and resonance quality factor enhancements, followed by a new readout method for noise reduction and signal-to-noise ratio (SNR) enhancement in actual wireless measurements [20]. Along with engineering specifications, design considerations are also focused on compatibility with suture-less minimally invasive surgical procedures in the sense of minimizing complexity and time of the corresponding operation for ease of device implantation in practice. Featuring parylene (poly-para-xylene) as the biocompatible polymeric material, the passive LC-based pressure sensor is designed to be completely implantable in the intraocular environment so that long-term continuous IOP monitoring of glaucoma patients using such telemetric system can be fulfilled.

\section{SySTEM DESCRIPTION}

\section{A. System Overview}

The concept of the wireless IOP sensing system in the passive electrical sensing scheme is shown in Fig. 1, and the associated wearable reader paradigm (e.g., glasses when the subject is awake and eye patch during his/her sleep) is proposed as shown in Fig. 2 for autonomous and continuous monitoring in practice. Because the IOP has insignificant dependence on the pressures outside the eye [21], its continuous monitoring can be anticipated with faithful readouts from the sensor implant. The sensor implant is designed to have an electrical LC-tank resonant circuit with a corresponding resonant frequency represented as

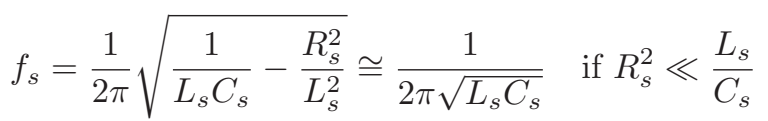

where $L_{s}, C_{s}$, and $R_{s}$ are the inductance, capacitance, and resistance of the sensor, respectively. By using an external coil to build an inductive coupling link with the implanted sensor, the equivalent impedance viewed from the measurement instrument can be derived using circuit analysis as [17], [22]

$$
Z_{\text {eq }}=\frac{V}{I}=j 2 \pi f L_{r}\left[1+k^{2} \frac{\left(\frac{f}{f_{s}}\right)^{2}}{1-\left(\frac{f}{f_{s}}\right)^{2}+\frac{1}{Q_{s}} j \frac{f}{f_{s}}}\right]
$$

where $V$ and $I$ are the exciting voltage and current across the reader coil, $f$ is the excitation frequency, $Q_{s}=$ $R_{s}^{-1}\left(L_{s} C_{s}^{-1}\right)^{1 / 2}$ is the quality factor of the sensor at resonance, and $k$ is the coupling coefficient of the inductive link (totally dependent on physical geometries such as the planar size of the sensor and reader coils, and the separation distance between the coils [15], [23]). Therefore, from (2), a phase-dip technique can be applied to wirelessly detect the resonant frequency of the sensor as the phase of the complex impedance $Z_{\text {eq }}$ drops to the minimum in the frequency scan from the measurement instrument, such as an impedance analyzer, network analyzer, or other involving customized electronics. When the sensor is excited at resonance, $Z_{\text {eq }}$ becomes

$$
Z_{\text {eq }}=j 2 \pi f_{s} L_{r}\left(1+j k^{2} Q_{s}\right)
$$

with minimized impedance phase, so the corresponding phase dip magnitude is maximized and can be approximated as

$$
\Delta \phi \cong \tan ^{-1}\left(k^{2} Q_{s}\right)
$$

The phase-dip magnitude determines the signal strength in the wireless sensing system and is dependent on $k$ and $Q_{s}$. Because of such relation between the impedance phase dip and the resonance frequency of the sensor, the latter can be identified if one can detect a phase dip in the frequency scan of the equivalent impedance. Now, if the sensor implant has pressure-sensitive electrical components, its resonant frequency will be shifted based on external pressure variation, thus registering the in situ environmental pressure. As a result, by finding the updated phase dip, the frequency shift can be obtained, and the corresponding pressure change can be analyzed. The involved electrical characteristic change can be interrogated using the external reader coil to accomplish continuous wireless IOP monitoring. Because the entire sensing scheme is based on impedance measurement given by (3), separate strategies can be applied to the sensor and reader as follows to increase the measurement sensitivity, leading to a more distinguished phase dip and correspondingly an enhanced sensing distance, of the overall sensing performance.

\section{B. Sensor Implant}

1) Design: In terms of sensor improvement than the previous work [19], both the quality factor and integrated coil size of the LC sensor should be greatly increased in order to increase the $k^{2} Q$ factor in (4). On the other hand, the entire device needs to be in a small form factor suitable in order to minimize associated surgical difficulty and complications during implantation. As a result, the proposed wireless IOP microsensor incorporates a flexible coil disk [17] to best meet 

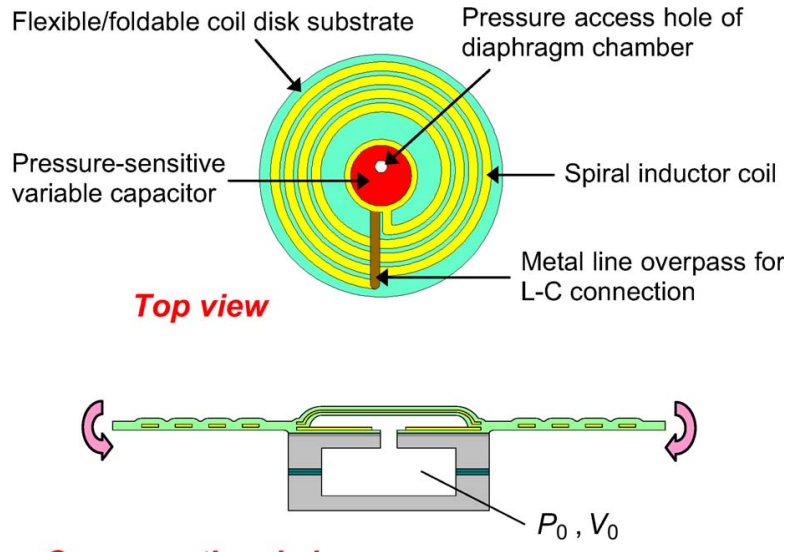

Cross-sectional view

Fig. 3. Pressure sensor design schematics with flexible/foldable coil disk substrate. The bottom rigid piece shown in cross-sectional view supports the deformable diaphragm as well as provides sealed cavity for reference in gauge pressure sensing.

both requirements. Fig. 3 shows the design of the microsensor comprising a pressure-sensitive parallel-plate variable capacitor embedded in a deformable diaphragm chamber, a spiral metal wire to serve as a planar inductor, and a flexible/foldable disk substrate to support the coil. The design particularly features a larger circular disk substrate to incorporate a larger spiral inductor. In the surgical design aspect, because the sensor is to be implanted in the anterior chamber of the eye, the diameter of the coil disk was correspondingly designed to be $4 \mathrm{~mm}$ to fit the iris rim width in normal conditions. This implant location has a tremendous advantage in aligning sensor and reader coils so that possible loss of signal strength through coil misalignment can be reduced. The coil disk substrate needs to be made out of a soft flexible material with sufficient mechanical flexibility so as to be folded to fit a small incision $(<2 \mathrm{~mm})$, for which the eye can spontaneously heal, toward suture-less minimally invasive device implantation. In the engineering aspect, the electrical characteristics of the sensor can be determined by using the established models [14], [23], [24], where the electrical inductance of such a circular spiral coil is calculated as

$$
L \cong \frac{\mu_{0} n^{2} d_{\mathrm{avg}} c_{1}}{2}\left[\ln \left(\frac{c_{2}}{F}\right)+c_{3} F+c_{4} F^{2}\right]
$$

where $n$ is the number of turns of the inductor, $d_{\text {avg }}$ is the averaged diameter of the coil windings, $F=\left(d_{\text {out }}-d_{\text {in }}\right) /\left(d_{\text {out }}+\right.$ $\left.d_{\text {in }}\right)$ is the fill factor of the coil windings, and $c_{1}-c_{4}$ are constant coefficients determined by the winding geometry. The electrical resistance is mainly contributed by the inductor wire which imperatively has a series resistance calculated, with consideration of the high-frequency skin effect, as

$$
R_{s}=\frac{\rho l}{w \delta\left(1-e^{-h / \delta}\right)}
$$

where $\rho$ is the electrical resistivity of the metal, $w$ and $h$ are the metal line width and height, respectively, and $\delta$ is the frequency-dependent metal skin depth given by

$$
\delta=\sqrt{\frac{\rho}{\pi f \mu}}
$$

with $\mu$ being the magnetic permeability of the metal. The electrical capacitance of the sensor can be expressed as

$$
C_{s}=C_{s, g}+C_{s, p}
$$

where $C_{s, g}$ is the capacitance determined by the integrated circular parallel metal plates with a gap separation at the center of the sensor and $C_{s, p}$ is the parasitic/stray capacitance generated by the other components in the entire device. As being embedded in the circular freestanding diaphragm at the center of the sensor, the parallel-plate capacitor is sensitive to pressure. When controlled in small deflection regime, depending on design parameters, the diaphragm deformation profile under a pressure loading can be described as

$$
w(r)=\frac{\Delta P a^{4}}{64 D}\left[1-\left(\frac{r}{a}\right)^{2}\right]^{2}
$$

where the deformation is dominated by the resistance of the diaphragm to bending [25] or

$$
w(r)=\frac{\Delta P a^{4}}{4 \sigma t}\left[1-\left(\frac{r}{a}\right)^{2}\right]
$$

where the deformation is dominated by the internal stress of the diaphragm [26]. In (9) and (10), $r$ is the radial coordinate from the center of the circular diaphragm, $\Delta P$ is the pressure difference across the diaphragm, $a$ is the diaphragm radius, $D=E t^{3} /\left[12\left(1-v^{2}\right)\right]$ is the flexural rigidity of the diaphragm in which $E$ and $v$ are Young's modulus and Poisson's ratio of the material, respectively, $t$ is the diaphragm thickness, and $\sigma$ is the internal stress of the diaphragm. This diaphragm deformation induces change of the gap between the plates and change of the corresponding equivalent capacitance. With incorporating such electrical-mechanical-coupled effect, the electrical characteristics and the pressure response of the LC sensor can be well designed.

In terms of sensor realization, the sensor is designed to be packaged with a rigid piece having air/gas cavity to provide pressure reference in gauge pressure sensing. The width of the backing piece is predetermined $1.5 \mathrm{~cm}$ so that the coiled disk can be folded to smaller than $4 \mathrm{~mm} \times 2 \mathrm{~mm}$ for sutureless intraocular implantation as well as for ease of realization and handling of the parallel-plate variable capacitor. Because this nonelectronic sealing piece is necessary for the LC sensor, surgical anchoring features for suture-less implant fixation on the iris of the eye are chosen to be created on this piece to avoid additional fabrication efforts on the sensor side. Overall, the form factor of the sensor implant must be substantially smaller than the space of the anterior chamber to ensure compatibility with natural eye reactions and motions. In addition, the implant has to be designed with smooth edges to prevent any possible scratch/cutting to the tissue during and after implantation. All involving materials in the sensor implant are in implantable grade. Parylene C (poly-chloro-p-xylylene) is selected as the diaphragm and disk substrate material because of its flexibility (Young's modulus $\sim 4 \mathrm{GPa}$ ), CMOS/MEMS process compatibility, and biocompatibility (USP Class VI grade) [27], [28]. 

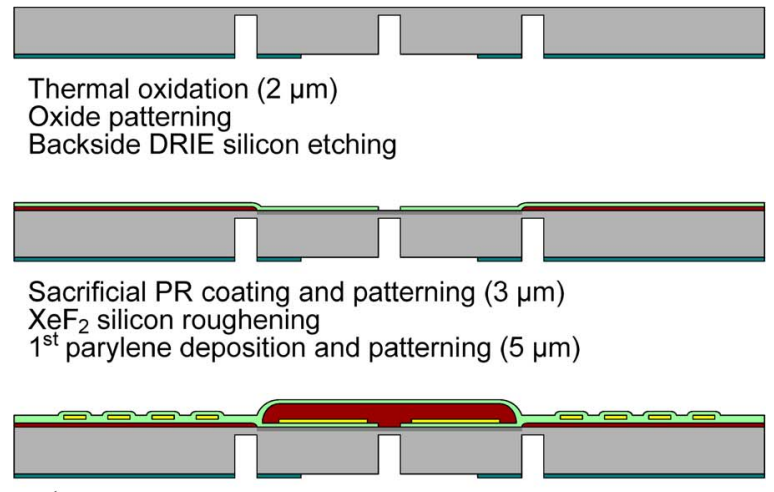

$1^{\text {st }}$ metal deposition and patterning $(3 \mu \mathrm{m})$

Sacrificial PR coating and patterning $(10 \mu \mathrm{m})$

$2^{\text {nd }}$ parylene deposition and patterning $(8 \mu \mathrm{m})$

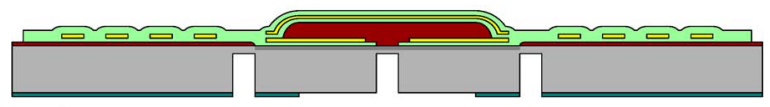

$2^{\text {nd }}$ metal deposition and patterning $(0.5 \mu \mathrm{m})$

$3^{\text {rd }}$ parylene deposition and patterning $(7 \mu \mathrm{m})$

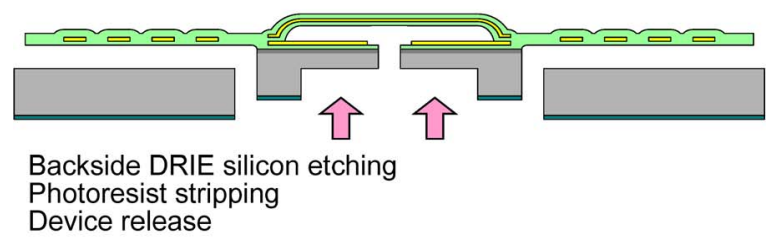

$\square$ Silicon $\square \mathrm{SiO}_{2} \square$ Photoresist $\square$ Parylene $\square \mathrm{Ti} / \mathrm{Au}$

Fig. 4. Fabrication process flow of modified flexible-coiled microsensor. The arrows indicate the outcome of the device release after microfabrication processes.

Additionally, its low water permeability $\left(0.08 \mathrm{~g} \cdot \mathrm{mm} / \mathrm{m}^{2} \cdot\right.$ day $)$ and low water absorption (less than $0.06 \%$ after $24 \mathrm{~h}$ ) favor stable behavior of such a device when immersed in the aqueous humor of the eye after implantation.

2) Fabrication: The fabrication process of the wireless pressure sensor is shown in Fig. 4 facilitated by lowtemperature multilayer parylene micromachining and deep silicon etching technologies. The fabrication processes started with thermally growing $2-\mu \mathrm{m}$ oxide on a double-side-polished silicon wafer. The oxide was patterned using buffered hydrofluoric acid (BHF, Transene Company Inc., Danvers, MA) with photoresist as a mask. AZ series photoresist (Clariant Corp., Charlotte, NC) was used in this step as well as the following steps throughout the described microfabrication processes. Deep reactive-ion etching (DRIE) in a PlasmaTherm SLR system (Unaxis Inc., St. Petersburg, FL) was performed on the backside of the wafer to define device release boundaries and access hole of the pressure diaphragm chamber until leaving approximately $50-\mu \mathrm{m}$ silicon for ease of through-wafer etching using the remaining oxide as the mask. The backside oxide was protected during DRIE to be used as a hard etch mask in the final through-wafer etch process. In front-side processes, a photoresist layer was created not only as a sacrificial layer for coil disk release but also as a mask to gas-phase xenon difluoride $\left(\mathrm{XeF}_{2}\right.$, Nesca Corp., Pretoria, South Africa) silicon roughening for physically strengthened parylene-silicon adhesion of the device [29]. Afterward, multiple parylene and metal
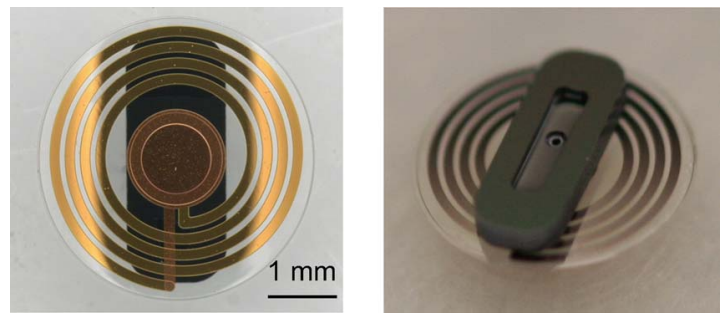

Fig. 5. Full-scale photographs of the microfabricated flexible-coiled wireless pressure sensor with its (left) top and (right) bottom views before being packaged. The diameter of the flexible coil disk was $4 \mathrm{~mm}$.

layers were deposited and patterned to create the flexible-coiled pressure sensor structures along with using another sacrificial photoresist layer to define the freestanding pressure diaphragm chamber. Parylene $\mathrm{C}$ deposition was conducted in a Cookson Electronics PDS 2010 system (Specialty Coating Systems Inc., Indianapolis, IN). The bottom parylene layer mechanically supports the metal coil windings and electrically isolates the other device layers from the substrate. The metals were e-beam evaporated for better material quality purposes, while thick deposition was required to obtain low overall resistance from the metal lines. Two thick titanium/gold layers $(200 \AA / 3 \mu \mathrm{m}$ and $200 \AA / 0.5 \mu \mathrm{m}$ ) were patterned using standard metal etching techniques. Twice thicker gold layers $(6$ and $1 \mu \mathrm{m})$ were also created in different samples for comparison in sensor testing. Titanium was involved to provide good adhesion between gold and parylene layers. Moreover, besides being used as the top parallel capacitor plate, the second metal layer was arranged to be with all boundaries of the parylene diaphragm chamber in order to effectively serve as a strong barrier to water vapor and gas transmissions/permeations in between the encapsulated air/gas and the ambience of the device [30]. This inclusion can effectively prevent the sensor implant from significant performance drift, which supports its long-term operation in the intraocular environment. In addition, in fact, this metal layer is extended to the surrounding of the diaphragm chamber to contribute additional capacitance by interaction with the underneath parylenemetal layers, which facilitates in controlling the device resonant frequency falling into the reader scan range of interest. Devices were finally released with a backside recess for larger closed volume of pressure reference after performing another DRIE followed by photoresist stripping with acetone. Fig. 5 shows the air-dried microfabricated sensor before device packaging.

The postmicrofabrication sensor packaging was conducted in air under atmospheric pressure at room temperature as the conditions of the encapsulated air. A nonelectronic piece was attached to the bottom of the microfabricated sensor using biocompatible (USP Class VI grade) epoxy to form an air cavity inside the device for pressure reference in gauge pressure sensing. The overall size was measured at $\phi 4 \mathrm{~mm} \times 1 \mathrm{~mm}$ and reduced to a form factor of $4 \mathrm{~mm} \times 1.5 \mathrm{~mm} \times 1 \mathrm{~mm}$ by folding the coil disk as shown in Fig. 6, suitable for minimally invasive intraocular implantation. Given the high yield strain $(\sim 3 \%)$ characteristic of parylene $\mathrm{C}$, the flexible disk can be stretched back to its original circular shape without severe permanent deformation or other damages after this extent of folding, and the inductor characteristics were hence unvaried. 

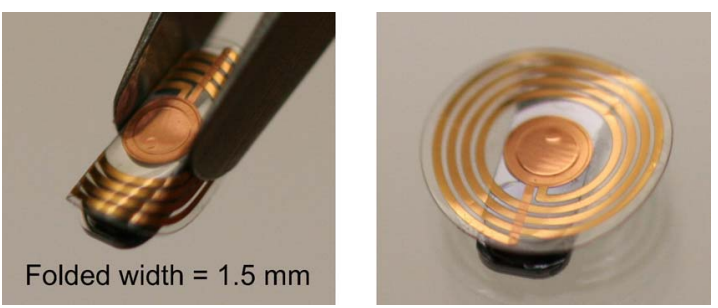

Fig. 6. Device flexibility demonstration. The device width could be reduced to $1.5 \mathrm{~mm}$ (predetermined by bottom silicon substrate) when folded (left), then stretched back after folding (right) without permanent deformation or other severe damage.

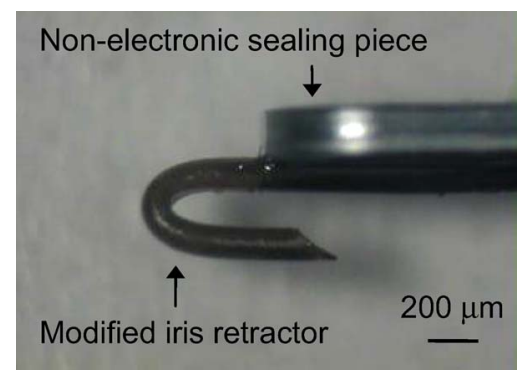

Fig. 7. (Side view) Representative iris anchor assembly result. The tapered retractor can hook the iris stratum to enable minimally invasive device fixation inside the eye.

In surgical aspect, a flexible ophthalmic iris retractor (Alcon/Grieshaber AG, Schaffhausen, Switzerland) was modified on-bench using a razor blade and attached to the nonelectronic sealing piece using biocompatible (USP Class VI grade) epoxy to serve as the tissue anchor for suture-less implant fixation. Fig. 7 shows the representative anchor assembly result. This surgical attachment and the microfabricated sensor were assembled as the final packaged device. After being introduced into the anterior chamber of the eye, the tapered feature at the end of the retractor hook penetrates the iris stratum to provide sufficient anchoring to the device in the implant location. This anchoring process is designed to be reversible in case the implant needs to be removed from the eye afterward. Only one single hook is used for the implant, or the iris would be anticipated with serious damage during pupil dilation/contraction. Nevertheless, using one hook was proven to provide strong fixation of the implant through rigorous vibration test and, afterward, animal studies. After packaging, the device can further be conformally coated by a thin parylene $\mathrm{C}$ layer to ensure its long-term biocompatibility in the intraocular environment.

\section{Readout Method}

Even though the modified sensor design was implemented, it is still not sufficient for achieving the required sensing distance when using the primitive readout method in the previous work [19] due to high noise from the actual testing system. Consequently, on the concept of phase-dip spectrum recovery for frequency recognition in further coil separation distance, a supportive data-processed external readout method was accordingly developed. This method only involves postmeasurement data processing and analysis, so that no additional efforts are needed to be made on the physical sensor or reader components in the measurement unit. The process scheme is based on a noise reduction concept with measurement sample averaging [31], accompanied by frequency-averaging procedure for better phase-dip curve recovery. First of all, multiple frequency scan samples were obtained and categorized to two different groups: baseline signal and sensor signal, depending on whether the reader coil was interrogating or interacting with the sensor implant. The necessary amount of sample data were captured and stored to the personal computer through a data acquisition system. Afterward, the data were processed in a series of operations, including the following: 1) baseline/background subtraction and 2) averaging of the processed scans (i.e., time averaging) in common signal processing principle. The baseline/background subtraction serves the purpose of eliminating substantial environmental interferences on the sensor-reader coil interaction, and the time averaging can significantly reduce the random noise of the signals from the actual sensor and reader coils. The sequence of such operations is interchangeable due to their linear operation nature, also resulting in the possibility of algorithm optimization depending on actual hardware/software constraints. The representative processed data through such operation are shown in Fig. 8. In this example, the measured phase noise was successfully reduced from the original value of $\sim 0.1^{\circ}$ to $\sim 0.01^{\circ}$ by differentiating the signals and further to $\sim 0.003^{\circ}$ after processing 20 scan samples (ten for the baseline signal and ten for the sensor signal), indicating more than $30 \times$ noise reduction so as to enhance the resultant sensing distance, even without signal strengthening by other modification on the sensor implant or the associated reader coil setup.

After such operation, the data after baseline/background subtraction and time-averaging operations can be investigated for detectable phase-dip frequency, as shown in Fig. 8(d). If a phase dip can be detected at a certain frequency point, the data are stored for the corresponding pressure measurement analysis. However, if not, further data processing such as frequency averaging [32] (which is demonstrated in this work) or phasedip curve fitting [17], [22] needs to be utilized to virtually increase the SNR of the measured data. For example, the data in Fig. 8(d) were further processed with appropriate frequency averaging to generate the plot as shown in Fig. 9 with enhanced SNR for more accurate phase-dip detection. Fig. 10 shows another successful data example benefited from the frequencyaveraging operation. These examples clearly illustrate the advantages of exploiting the proposed data processing method. In practice, all the aforementioned readout and postprocessing procedures should be completed in a short time period, so that sensing result drift or deviation due to interferences to the test objects offset can be minimized during measurements. Combining the modified implantable flexible-coiled sensor and the data-processed external readout method, prevailing lownoise long-range signal detection can be anticipated to facilitate the continuous wireless passive pressure sensing system.

\section{Results AND Discussion}

The microfabricated devices were tested both on-bench and in vivo to characterize their electrical, physical, and 


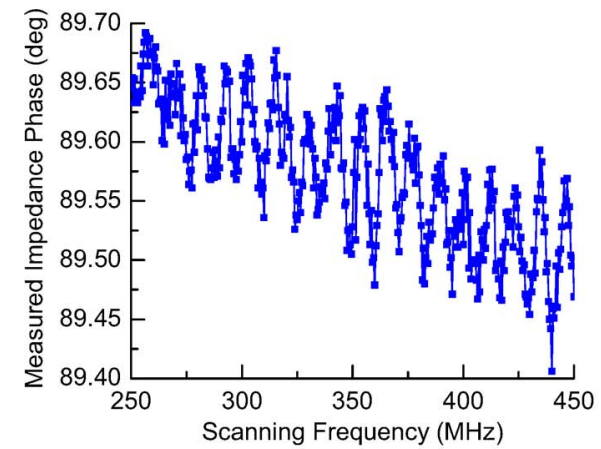

(a)

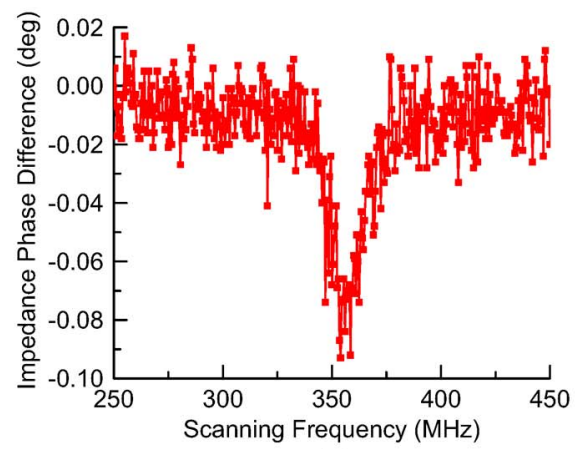

(c)

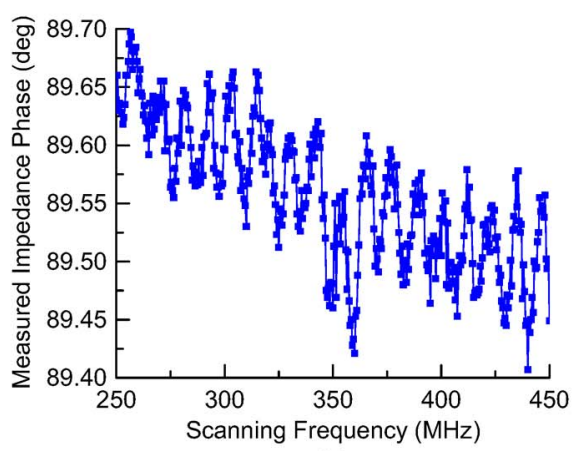

(b)

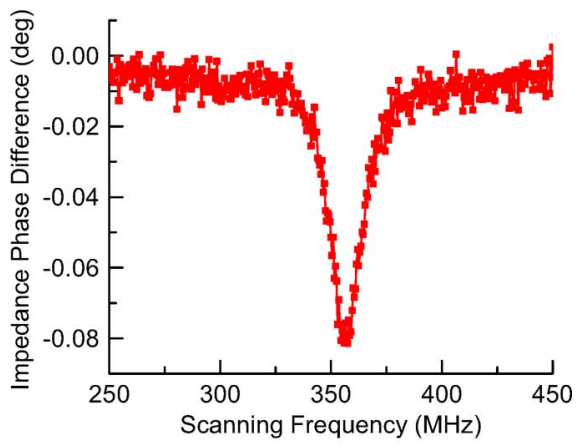

(d)

Fig. 8. Data samples through signal processing operation as example. (a) Unprocessed baseline signal. (b) Unprocessed sensor signal. (c) Processed signal after baseline/background subtraction. (d) Processed signal after time averaging. The number of total sample size is 20 [ten each in (a) and (b)].

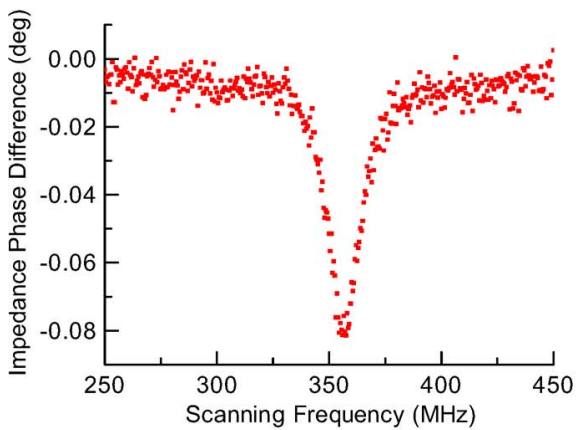

(a)

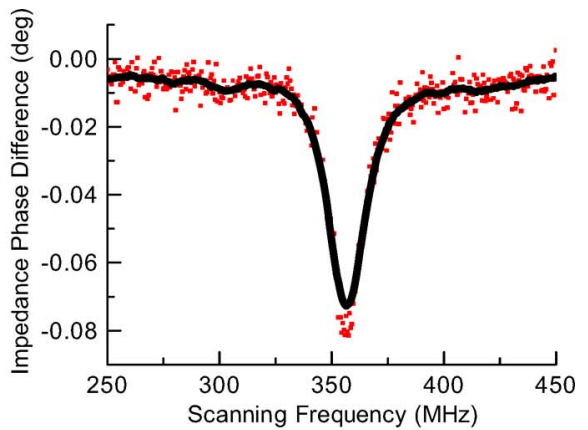

(b)

Fig. 9. Frequency averaging with data samples used in Fig. 8 to illustrate the enhanced phase-dip detection scheme. (a) Data points from Fig. 8(d) before frequency averaging. (b) Smoothed curve after frequency averaging, which leads to higher SNR and, thus, more obvious phase dip over the entire frequency spectrum.

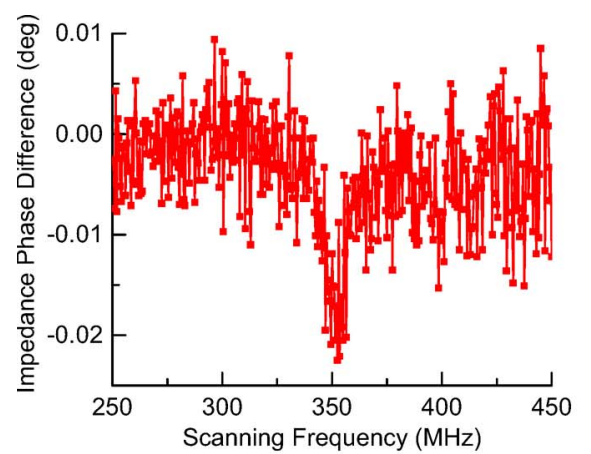

(a)

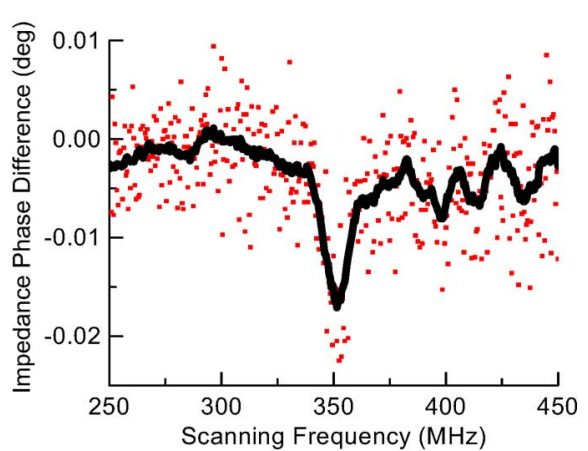

(b)

Fig. 10. Another data sample (sample size $=20$ ) used to illustrate the frequency-averaging operation. (a) Processed data before frequency averaging. (b) Smoothed curve after frequency averaging with unveiled impedance phase dip. 
TABLE I

Measured Electrical Parameters of the Microfabricated FleXible-CoIled Wireless PRESSURE SENSOR

\begin{tabular}{cc}
\hline Parameters & Values \\
\hline Inductance & $57 \mathrm{nH}$ \\
Capacitance & $3.6 \mathrm{pF}$ \\
Resistance at resonance & $4.2 \Omega$ \\
Resonant frequency & $\sim 350 \mathrm{MHz}$ \\
Quality factor & $\sim 30$ \\
\hline
\end{tabular}

surgical/biological behaviors. During testing, an HP 4195A network/spectrum analyzer (HP/Agilent Technologies Inc., Santa Clara, CA) under standard settings was utilized in connection with hand-wound coils made out of standard insulated copper wire to serve as the external reader for wireless sensing demonstration. Measured equivalent impedance spectra data were stored in a personal computer using the LabVIEW program (National Instruments Corp., Austin, TX) for postprocessing and analysis demonstration. Testing results are described in the following sections.

\section{A. On-Bench Characterization}

Electrical parameters of the sensors were first obtained by measuring the actual devices as well as several testing structures. Table I lists the experimental results in good agreement with the theoretical calculations. The higher resistance at resonance was resulted from the noticeably small metal skin depth at high frequency $(\sim 4.2 \mu \mathrm{m}$ for gold at $350 \mathrm{MHz})$. The resonant frequency of the LC sensor was determined where the phase of the impedance $Z_{\text {eq }}$ dipped to the minimum at the frequency $f_{\min }$, which is highly correlated to $f_{s}$ and can be expressed as [21]

$$
f_{\min }=f_{s}\left(1+\frac{k^{2}}{4}+\frac{1}{8 Q^{2}}\right)
$$

while the discrepancy between the two was able to be controlled within $0.1 \%$ (particularly less than $200 \mathrm{ppm}$ when conducting wireless pressure sensing tests) given the negligible terms contributed by $k$ and $Q$ in this work.

A customized pressure control configuration, as shown in Fig. 11, was utilized for wireless pressure sensing demonstration. The device was placed inside a chamber connected to a pressurization setup where a commercial off-chip pressure regulator and a pressure gauge were used to provide $0.5-\mathrm{mmHg}$ tuning resolution. Accurate environmental pressure variations could thus be created for the device $\left(\Delta P=P_{\text {outside sensor }}-\right.$ $P_{\text {inside sensor }}$ ) with this pressure control setup.

Although the pressure sensitivity was expected to be small from the device design, the sensing was compensated by the high sensor resonant frequency to reach reasonable pressure responsivity for detection of phase-dip shift with respect to environmental pressure variations. Measured phase-dip curves were obtained as shown in Fig. 12 to characterize the sensor

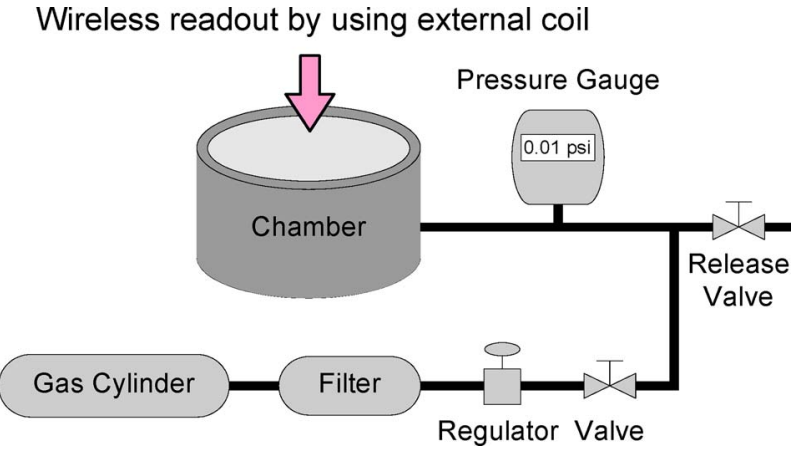

Fig. 11. Schematic of the on-bench pressure testing setup for characterization of the packaged flexible-coiled sensor.

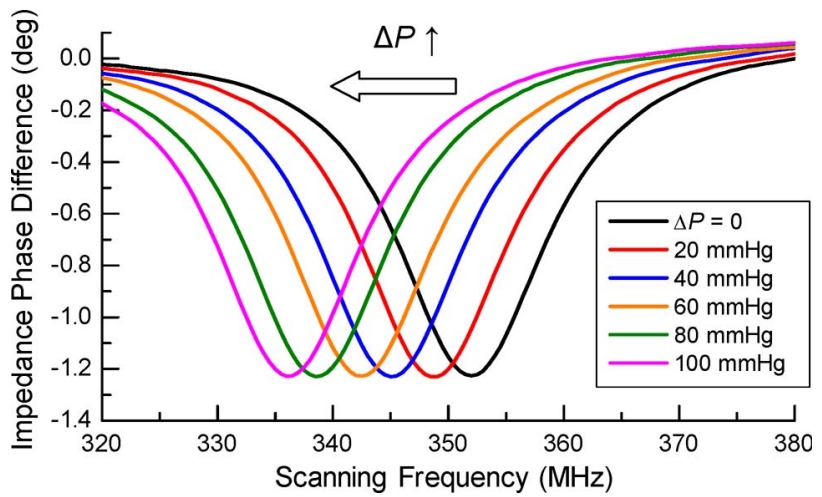

Fig. 12. Overlay plot of the measured phase-dip curves of the sensor in onbench wireless pressure sensing test. $\Delta P=P_{\text {outside sensor }}-P_{\text {inside sensor }}$.

behavior. The pressure response of the sensor can be modeled as follows with previous derivation in [19]. First of all, the normalized shifted phase-dip frequency of the sensor can be written as

$$
\begin{aligned}
\frac{f_{\min }(\Delta P)}{f_{\min }(\Delta P=0)} & =\frac{\frac{1}{2 \pi \sqrt{L_{s}\left(C_{s}+\Delta C_{s}\right)}}}{\frac{1}{2 \pi \sqrt{L_{s} C_{s}}}} \\
& =\sqrt{1-\frac{\Delta C_{s}}{C_{s}}}, \quad \text { if } \Delta C_{s} \ll C_{s}
\end{aligned}
$$

where $\Delta C_{s}$ is the increased electrical capacitance from the pressed diaphragm by increased environmental pressure applied on the sensor, and it is known to be proportional to diaphragm deflection (inversely proportional to the gap between the parallel capacitor plates). As a result, when controlling the diaphragm deformation in small deflection regime where the profile is given in either (9) or (10), it can be derived from (12) as

$$
\frac{f_{\min }(\Delta P)}{f_{\min }(\Delta P=0)}=(1-\alpha \Delta P)^{1 / 2}
$$

where $\alpha$ is a parameter incorporating the mechanical behavior of the diaphragm. This relationship was used to characterize the pressure sensitivity of the packaged sensor as shown in Fig. 13 in all wireless pressure sensing tests. Furthermore, it was also observed during sensor characterization that the volume change effect from the encapsulated air/gas reference [19] was not negligible in this work. As shown in Fig. 14, the 


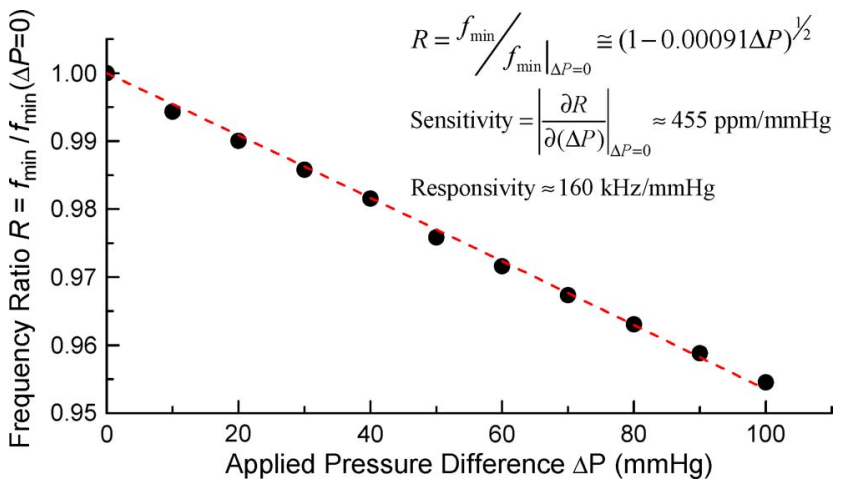

Fig. 13. Characterization of pressure response of the sensor.

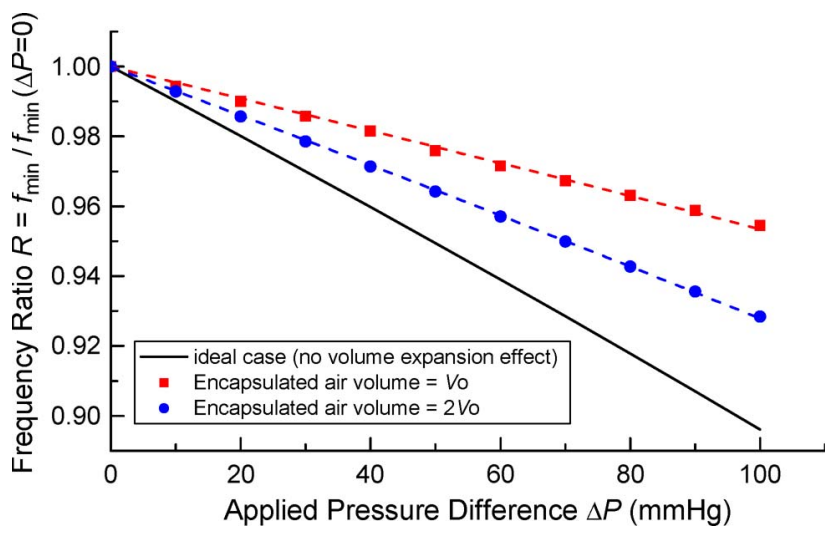

Fig. 14. Characterization of volume change effect of the encapsulated air in the sensor.

pressure sensitivity of the theoretical model was designed to be $985 \mathrm{ppm} / \mathrm{mmHg}$, while in measurements, only $455 \mathrm{ppm} / \mathrm{mmHg}$ sensitivity was obtained when the sealed cavity volume was $500 \mu \mathrm{m} \times 2000 \mu \mathrm{m} \times 250 \mu \mathrm{m}$ (width $\times$ length $\times$ height). By designing a different geometry on the nonelectronic sealing piece to double the sealed cavity volume $(500 \mu \mathrm{m} \times$ $2000 \mu \mathrm{m} \times 500 \mu \mathrm{m})$ in the final packaged device, the pressure sensitivity and responsivity were experimentally characterized to be $695 \mathrm{ppm} / \mathrm{mmHg}$ and $243 \mathrm{kHz} / \mathrm{mmHg}$, approximately $1.52 \times$ enhancement over the original values and closer to the ideal values. These results were in good agreement with expectations after incorporating the ideal gas law and showed the possibility of altering/optimizing the design parameters depending on actual application specifications.

Next, the data-processed readout method was exploited for sensing distance studies of such a wireless system. For demonstration purposes, devices with different e-beam evaporated gold thicknesses $(3 \mu \mathrm{m} / 0.5 \mu \mathrm{m}$ and $6 \mu \mathrm{m} / 1 \mu \mathrm{m}$ for first and second metal layer structures, respectively) were prepared. The doubled metal thickness resulted in approximately 1.5 times $Q_{s}$ enhancement (from $\sim 30$ to $\sim 45$ ). This enhancement was primarily limited by the high-frequency skin depth effect from the inductor wire resistance at the operating frequency. It is also worth noting that the metal wire thickness could be further increased by depositing a thicker metal through the described evaporation method, an electroplating alternative, or by stacking using a special postmicrofabrication technique [33] to fur- ther reduce the series resistance of the inductor and enhance the overall quality factor. The necessary number of samples for averaging operations depends on the final frequency measurement resolution needed for ultimate pressure sensing. The frequency noise (i.e., frequency fluctuation) in measurements needs to be lower than the resultant frequency shift from environmental pressure variation for reliable pressure sensing accuracy. As previously stated, the pressure sensitivity of the sensor was characterized to be approximately $455 \mathrm{ppm} / \mathrm{mmHg}$, which means that the allowed frequency fluctuation must be lower to achieve a $1-\mathrm{mmHg}$ pressure sensing accuracy. With this information, experiments were designed to verify the sample size effect on SNR and frequency fluctuation. By using a $Q_{s} \sim$ 45 sensor with a 4-mm-diameter on-sensor coil interrogated by a 15-mm-diameter hand-wound reader coil at 2-cm separation distance, the results are shown in Fig. 15(a) with the SNR defined as

$$
S N R=\frac{\Delta \phi_{\max }}{\Delta \phi_{\text {noise }}}
$$

where $\Delta \phi_{\max }$ is the maximum impedance phase dip throughout the frequency scan and $\Delta \phi_{\text {noise }}$ is the noise from the measurement system. More than 80 measurement samples had to be collected in order to justify $S N R>10$ and the associated frequency shift resolution smaller than $400 \mathrm{ppm}$, which is less than that by $1-\mathrm{mmHg}$ pressure difference. Fewer samples could be collected, but the overall pressure accuracy would be reduced. On the other hand, more samples could be collected, but the processing capacity and time would need to be considered. This tradeoff ultimately defines the sampling rate specification of readouts in practice. In addition, the noise limit in the measurement system jointly determines the size of data to be collected and analyzed. Fig. 15(b) shows that the achieved $\Delta \phi_{\text {noise }}$ was lower than $0.0015^{\circ}$ by processing more than 80 samples (40 for sensor signal and 40 for baseline signal) and approaching the limit $\left(\sim 0.001^{\circ}\right)$ when more than 200 samples were collected. The fitted noise decay trend was well predicted with the assumption of involving uncorrelated noises in individual measurements. The noise limit, which could not be further cancelled or suppressed, represents the "white noise" in the measurement system. Improvements on reader unit (e.g., the use of low-noise electronics) and inductive coupling link would be necessary to fundamentally decrease the noise limit and thus achieve higher SNR and longer range sensing.

For wireless pressure sensing using the flexible-coiled sensor and the data-processed external readout method in this work, the criterion of determining the maximum sensing distance was set to be $S N R>10$ given the minimal $\Delta \phi_{\text {noise }} \sim 0.001^{\circ}$ so that the phase-dip frequency measurements were capable of resolving 1-mmHg pressure difference. The maximum sensing distance was therefore characterized to be 2 and $2.5 \mathrm{~cm}$ for $Q_{s} \sim 30(3-\mu \mathrm{m} / 0.5-\mu \mathrm{m}$ metal structures $)$ and $Q_{s} \sim 45$ (6- $\mu \mathrm{m} / 1-\mu \mathrm{m}$ metal structures) sensors, respectively. Experiments using a $Q_{s} \sim 45$ sensor with approximately 4-mmdiameter on-sensor coil interrogated by hand-wound reader coils in different sizes were conducted to further study the relation between the coil separation distance and the associated 


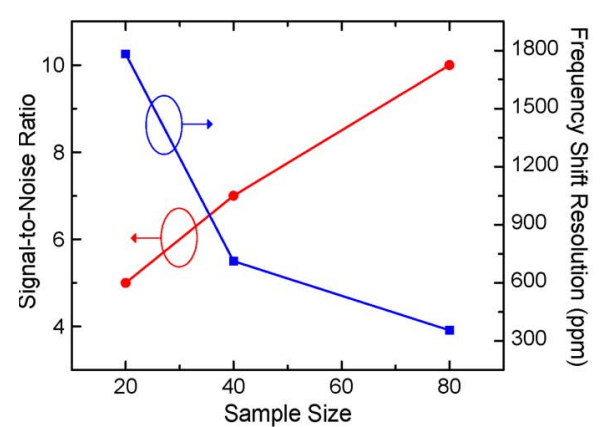

(a)

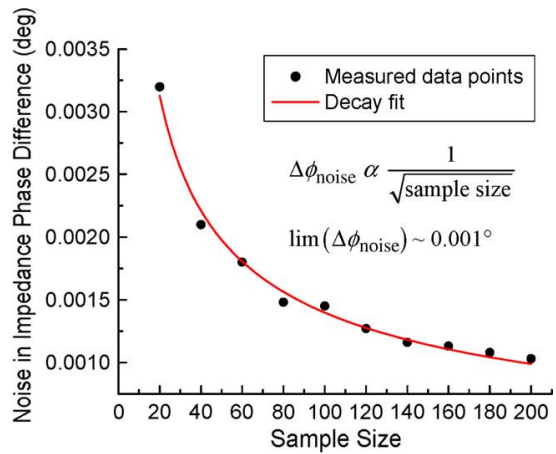

(b)

Fig. 15. Sample size effect on frequency measurements. (a) Obtained SNR and frequency shift resolution. (b) Obtained $\Delta \phi_{\text {noise }}$ with the exponential fit illustrating the reduction limit of using the data-processing readout method. Half of the samples were collected from the sensor signal, and the other half were from the baseline/background signal.

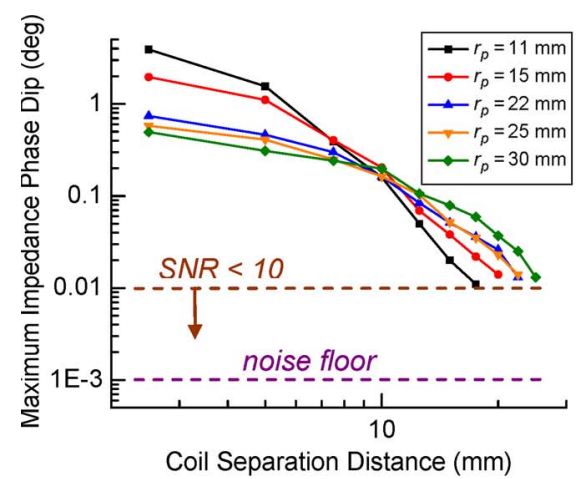

(a)

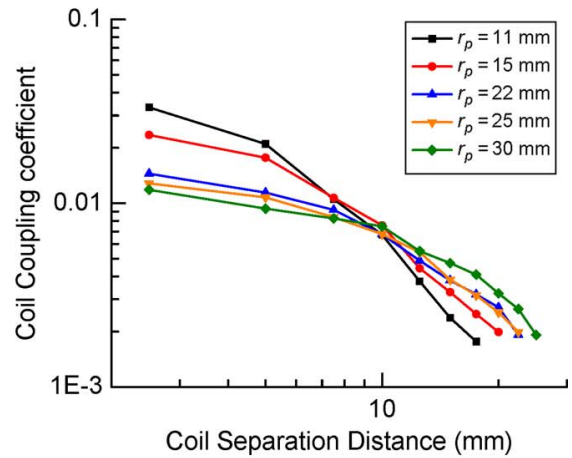

(b)

Fig. 16. Sensing distance study using $Q_{s} \sim 45$ sensor with approximately 4-mm-diameter on-sensor coil. (a) Measured maximum phase-dip plot. (b) Derived coupling coefficient plot using (4).

electrical performance. Fig. 16 clearly shows that the phase dip and the derived coupling coefficient from (4) decrease as the coil separation increases, with an empirical relation equivalent to that found in [31] as in

$$
\begin{cases}k \alpha z^{-1}, & \text { when } z \ll r_{p} \\ k \alpha z^{-3}, & \text { when } z \rightarrow r_{p}\end{cases}
$$

where $z$ is the coil separation distance and $r_{p}$ is the reader coil radius. Note that the separation distance in the experiments did not take into account angular misalignment between the coils, so effective distance needs to be addressed in reality. The obtained phase-dip-distance product analogous to gain-bandwidth product was higher for larger reader coils. Both the near-field and far-field coil coupling situations were well described by (15) and were in good agreements with theoretical expectations [14], [31], [34]-[36]. With these findings, the ultimate reader coil can be properly designed and realized as part of the glasses-type reader shown in Fig. 2, incorporating the necessary glasses-to-iris distance $(1.5-2 \mathrm{~cm})$ in practical wireless sensing. The demonstration using the sensor prototype hence verifies the feasibility of modifying sensor parameters to increase the sensing distance for the required IOP monitoring specifications.

On-bench experimental studies also indicated that the sensor-reader coil coupling is substantially influenced by the

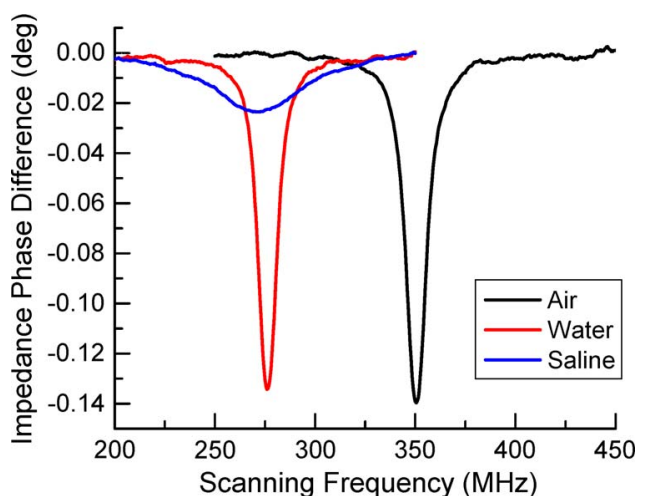

Fig. 17. Influence of interaction between sensor and different wavetransmission media.

medium in which the sensor is placed. As shown in Fig. 17, the phase-dip frequency was varied from $350 \mathrm{MHz}$ in air to approximately $275 \mathrm{MHz}$ in liquid (water and $0.9 \%$ saline) environments, approximately $29 \%$ difference. This frequency shift is due to the higher permittivity of water-based solutions (dielectric constant $\varepsilon_{r} \sim 80$ at $20{ }^{\circ} \mathrm{C}$ ) than that of air $\left(\varepsilon_{r} \sim 1\right)$. It causes an increased electrical field concentration on the medium and so virtually increases the total equivalent capacitance and decreases the resultant phase-dip frequency measured from the external reader. Furthermore, for lossy medium such 
as saline, the permittivity can be written in the complex form as [36]-[38]

$$
\varepsilon=\varepsilon^{\prime}-j \varepsilon^{\prime \prime}
$$

with the loss tangent defined as the ratio $\varepsilon^{\prime \prime} / \varepsilon^{\prime}$, a measure of the power loss in the medium. Different loss tangent values of water $(\sim 0)$ and $0.9 \%$ saline $(\sim 0.2)$ reflect their different lossy extent as media degrading the electromagnetic coupling of the coils. As a result, the measured quality factor was around the same value $\left(Q_{s} \sim 45\right)$ when the sensor was in water as compared with air, while it dropped to $\sim 6$ when the sensor was in saline. The incorporated sensing distances in water and saline were characterized as 2.5 and $1.5 \mathrm{~cm}$, respectively. Solutions to overcoming the decreased sensing distance in saline that has similar composition and properties to the aqueous humor need to be developed, despite the fact that $1.5 \mathrm{~cm}$ could be sufficient for practical IOP monitoring. Future works will be investigations on reducing the lossy medium effect. One possible solution that has already been demonstrated to be viable is strengthening the electrical isolation between sensor structures and the environment [17]. Preliminary test results suggested that $Q$ could be substantially recovered back to higher than 20 with covering $10-\mu \mathrm{m}$ parylene $\mathrm{C}$ on the inductor wires [39]. Nevertheless, the device pressure sensitivity was found invariant to different surrounding media.

Because the operation principle of such a sensor involves encapsulated air/gas reference, the corresponding phase-dip frequency drift due to possible changes of reference needs to be studied as well to verify the senor performance. The temperature sensitivity was characterized on-bench as approximately $1064 \mathrm{ppm} /{ }^{\circ} \mathrm{C}$, in good agreement with design expectations, considering a closed-system air/gas expansion effect based on the ideal gas law. The overall pressure accuracy obtained from this sensor was therefore approximately $2.5 \mathrm{mmHg}$, incorporating $\pm 1{ }^{\circ} \mathrm{C}$ temperature fluctuation in the implantation environment (i.e., inside the human body). Other than using temperature compensation techniques, the air/gas reference inside the sensor could be sealed either under low pressure (ultimately in vacuum as in absolute pressure sensors) or at high temperature so as to directly reduce the pressure-temperature correlation effect. The encapsulated air/gas could also be replaced by liquids to eliminate such an effect. It will be investigated in future studies that, after implementing these concepts in device packaging, the pressure sensing accuracy could potentially be improved to $1 \mathrm{mmHg}$ as promised for practical IOP monitoring. Soak tests showed that the phasedip frequency drift was less than $500 \mathrm{ppm}$, equivalent to the result by $1-\mathrm{mmHg}$ pressure variation, when the sensor was submerged in saline more than $100 \mathrm{~h}$ at both $25^{\circ} \mathrm{C}$ and $37^{\circ} \mathrm{C}$, confirming the effectiveness of parylene-metal-parylene isolation to liquid/gas permeation and absorption that could affect the pressure reference cavity and other sensor structures [17]. Table II summarizes the important characteristics of the microfabricated flexible-coiled wireless pressure sensor in this work. With the improvements shown here along the way after the developments of the first version of the device, future works
TABLE II

Measured Characteristics of THE PACKAGEd FLEXIBLE-COILED WIRELESS PRESSURE SENSOR

\begin{tabular}{|c|c|}
\hline Parameters & Values \\
\hline Planar dimensions & $\begin{array}{l}\text { (normal) } 4 \mathrm{~mm} \text { diameter } \\
\text { (folded) } 4 \mathrm{~mm} \times 1.5 \mathrm{~mm}\end{array}$ \\
\hline Resonant frequency & $\sim 350 \mathrm{MHz}$ \\
\hline Quality factor & $\sim 30 / \sim 45^{*}$ (in air) \\
\hline Sensing distance & $2 \mathrm{~cm} / 2.5 \mathrm{~cm}^{*}$ (in air) \\
\hline Pressure sensitivity & $455 \mathrm{ppm} / \mathrm{mmHg}^{\dagger}$ \\
\hline Pressure responsivity & $160 \mathrm{kHz} / \mathrm{mmHg}^{\dagger}$ \\
\hline Temperature sensitivity & $1064 \mathrm{ppm} /{ }^{\circ} \mathrm{C}$ \\
\hline $\begin{array}{l}\text { Resonant frequency drift } \\
\text { in saline environment }\end{array}$ & $\begin{array}{l}<500 \mathrm{ppm} \text { after } 100 \mathrm{hr} \\
\quad\left(\text { at } 25^{\circ} \mathrm{C} \text { and } 37^{\circ} \mathrm{C} \text { ) }\right.\end{array}$ \\
\hline $\begin{array}{l}\text { Projected pressure accuracy } \\
\text { in practical IOP monitoring }\end{array}$ & $2.5 \mathrm{mmHg}^{\ddagger}$ \\
\hline
\end{tabular}

on the engineering side will be focused on enhancing pressure accuracy, sensing distance, and device reliability.

\section{B. In Vivo Test}

The LC sensor packaged as described previously was used to conduct the in vivo test. Device implantation was managed using live rabbit eyes as the model to evaluate the feasibility of the sensor implant. The fully packaged device sealed with atmospheric air for pressure reference was conformally coated by a thin parylene $C$ layer to ensure its biocompatibility in the intraocular environment and then sterilized using ethylene oxide gas prior to the implantation surgery. All animal procedures in this study conformed to the ARVO Statement on the Use of Animals in Ophthalmic and Vision Research. Viscoelastic gel could be used to facilitate the device implantation. The implant was manipulated mainly with surgical forceps. The entire surgery was completed within 15 min because of its minimally invasive $(<2$-mm corneal incision using angled razor blade) nature, which minimizes surgical and postoperative complications. As shown in Fig. 18(a), the implant with folded coil disk was introduced into the anterior chamber of the eye through the incision using standard medical forceps. After the coil disk was stretched back, the implant was manipulated to be anchored on the iris using forceps under compatible surgical procedures without enlarging the incision. No sutures were required to this short thin incision as the eye could spontaneously heal for it. Fig. 18(b) shows the implant location in the intraocular environment after surgery. Follow-up study results, including fluorescein angiography and fundus photograph as shown in Fig. 19, confirmed that no device dislocation or postoperative complications, including inflammatory response or tissue encapsulation/fibrosis, were found over six months. This result 


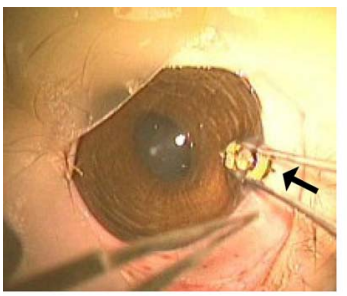

(a)

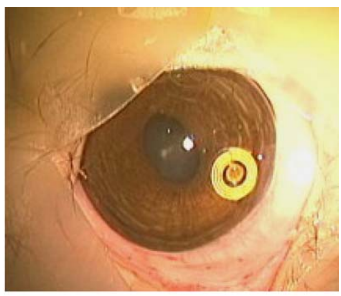

(b)
Fig. 18. Implantation surgery using live rabbit eye model. (a) Device being inserted into the anterior chamber through corneal incision. The arrow indicates the iris anchor on the backside of the flexible-coiled pressure sensor. (b) Implanted device in the intraocular environment as observed through the cornea.

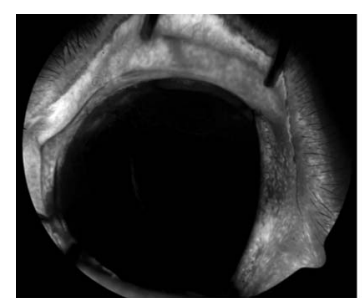

(a)

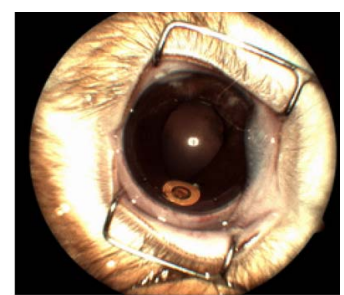

(b)
Fig. 19. Follow-up study results of in vivo device testing using rabbit model to verify bioefficacy and biocompatibility of the sensor implant. (a) Fluorescein angiogram showing no noticeable blood vessel leakage at the implantation site one week after surgery. (b) Fundus photograph showing no device dislocation or postoperative complications appeared in the intraocular environment six months after surgery. The implant was manipulated without dislocation for visual examination.

suggested no protein aggregation or other biofouling incidents, thus verifying the surgical/biological feasibility of using such implant paradigm with appropriate anchoring mechanism in the intraocular environment. In the physical aspect, no coil disk curling of the sensor implant was observed based on the fact of insignificant stress induced by the aqueous humor fluid in the anterior chamber. Pupil dilation/contraction could be a concern that the implant can possibly block the subject's sight to some extent, so a modified device topology and/or implantation scheme might be needed to address this issue when managing human's eye in the future.

In order to demonstrate the in vivo wireless pressure sensing feasibility of such a flexible-coiled sensor paradigm, acute animal testing using live rabbit eye models was conducted with an experimental setup as shown in Fig. 20. The obtained phase-dip curves were much clearer and more appropriate for pressure analysis as compared with those in previous work [19], attributable to higher SNR using the sensor and the developed data-processed external readout method. Although limited by the pressure control and monitoring scheme in surgical protocols at the moment of experiment such that no pressure measurement analysis could be performed, the shifted phasedip curves correlated to a qualitatively increased IOP in the model and markedly justified the possibility of the developed implant for wireless pressure sensing.

Given the results in the acute in vivo testing, an improved and more quantitative study was completed using an enucleated porcine eye as the animal model to characterize the pressure response of the sensor in the simulated intraocular environment.
An intraocular manometer was used with the infusion-based pressurization setup in this experiment to obtain precise IOP measurements as compared with the readouts from the sensor. The results shown in Fig. 21 were analyzed and confirmed in good agreement with the on-bench results shown in Fig. 12, indicating that the physical pressure response of the developed sensor was consistent when situated in different environments, regardless of the different electrical readouts due to the aforementioned medium effect. Extensive animal studies, including acute and chronic tests, along with the knowledge and the corresponding sensor improvement on overcoming the medium effect, are still underway in order to completely characterize the in vivo performance of the flexible-coiled wireless pressure sensor paradigm. Advanced surgical protocols and tools suitable for such an implant paradigm will also be investigated to better meet the requirements. After such improvements, tonometry or other noninvasive techniques will be used for comparison, as needed to replace the intraocular manometer in order to characterize and calibrate the sensor performance after implantation in practice.

Future work will also be focused on reader optimization and actual implementation. Development of a low-noise measurement system will be the first keystone. As described previously, besides improvements from using the developed dataprocessing-based readout method from a software perspective, low-noise circuitry and techniques from a hardware perspective should be included to electronically reduce the measurement noise and enhance the SNR in phase-dip frequency detection [31]. Other readout methods have also been explored in different research groups [40]-[42] with the aim of long-range telemetric sensing and are valuable for comparison to the configuration proposed in this work. Moreover, in cases where either the sensor-reader coil coupling or quality factor of the sensor is substantial enough to influence the frequency shift resolution based on (10), some compensation method would be required as part of the final telemetric system. For example, a distancecompensated technique [43] can be applied under this concept to obtain correct frequency values regardless of possible out-ofplane positional variation between the glasses (reader) and the iris (sensor), which is valuable for further study. These developed techniques involving additional electrical components or microelectronics implementation might be incorporated into the current readout method and will be extensively studied in the future. Eventually, the readout and postprocessing methods will be realized in a standalone portable system for measurement convenience. After such successful reader development, this wireless implant-based pressure sensing approach will be complete for practical continuous IOP monitoring and glaucoma study. Extensive comparisons between this approach and gold standard tonometry techniques will be given when sophisticated experimental setup and reader are available for long-term in vivo pressure monitoring tests.

\section{CONCLusion}

An implant-based wireless pressure sensing paradigm using electrical readout methodology has been successfully developed as a solution to continuous IOP monitoring. Development 


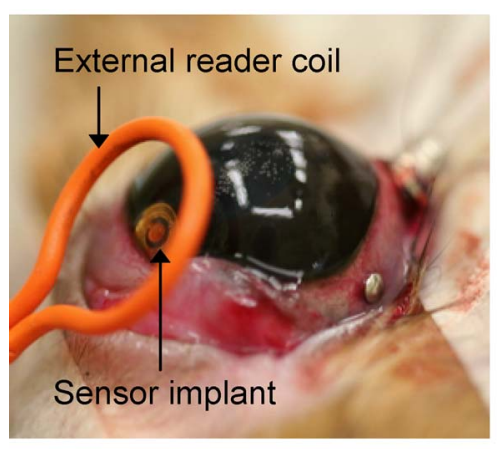

(a)

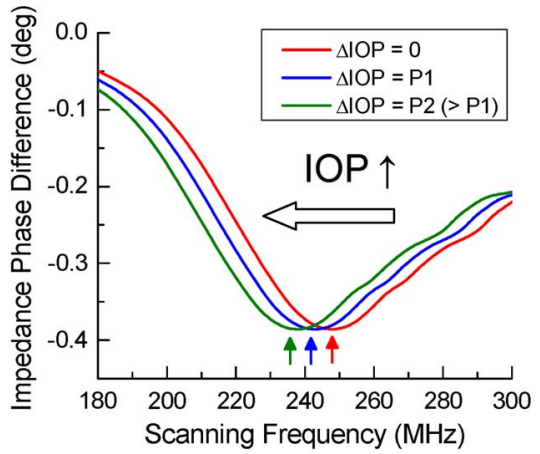

(b)

Fig. 20. Acute in vivo wireless pressure sensing test using rabbit model. (a) Photograph of the experimental setup. (b) Overlay plot of the measured phase-dip curves.

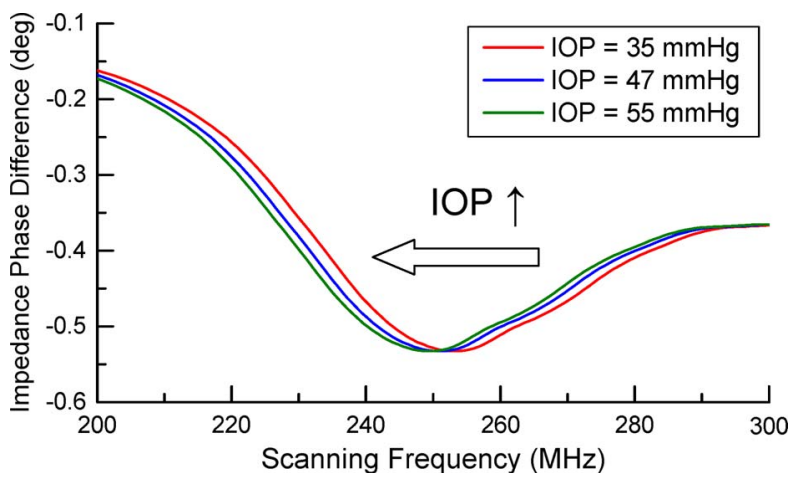

Fig. 21. Acute ex vivo wireless pressure sensing test using enucleated porcine eye model.

of both sensor implant and readout method has been addressed to accomplish practical wireless pressure sensing in a foreseeable glasses-reader paradigm. Complete design, fabrication, packaging, and characterization of the passive sensor incorporating a pressure-sensitive electrical LC-tank resonant circuitry are described. The LC sensor features a flexible/foldable inductor coil disk to achieve not only a long sensing distance through stronger inductive coupling with the external reader coil but also a small physical form factor, with an associated corneal incision smaller than $2 \mathrm{~mm}$, when the implant is introduced into the anterior chamber of the eye. Other surgical features were also included to facilitate the sensor suitable for minimally invasive suture-less intraocular implantation. Low-temperature multilayer polymer/metal micromachining technology was exploited to enable integrated sensor components fabricated on a monolithic substrate without the need for a multiwafer bonding process. A supportive data-processed external readout method was implemented to analyze the pressure measurements with enhanced resolution and distance. Engineering performance of the sensor-reader system and surgical/biological compatibility and efficacy of the sensor implant were characterized in a complete suite of on-bench, ex vivo, and in vivo experiments, with the results shown to be in good agreement with design parameters and meeting practical requirements. With successful demonstration and future implementation of the proposed sensor/reader improvement strategies, this wireless pressure sensing paradigm has great potential for fulfilling continuous, real-time, reliable, and convenient IOP monitoring in glaucoma patients.

\section{ACKNOWLEDGMENT}

The authors would like to thank W. Li for her assistance on data acquisition of electrical impedance measurements, D. C. Rodger for his assistance on surgical procedures and pressure sensing tests using animal models, and T. Roper for his assistance on sensor fabrication.

\section{REFERENCES}

[1] M. Sherwood, Ophthalmology, M. Yanoff and J. S. Duker, Eds., 2nd ed. St. Louis, MO: Mosby, 2004, pp. 1413-1473.

[2] S. Asrani, R. Zeimer, J. Wilensky, D. Gieser, S. Vitale, and K. Lindenmuth, "Large diurnal fluctuations in intraocular pressure are an independent risk factor in patients with glaucoma," J. Glaucoma, vol. 9, no. 2, pp. 134-142, Apr. 2000.

[3] E. Hughes, P. Spry, and J. Diamond, "24-hour monitoring of intraocular pressure in glaucoma management: A retrospective review," J. Glaucoma, vol. 12, no. 3, pp. 232-236, Jun. 2003.

[4] R. Puers, G. Vandevoorde, and D. D. Bruyker, "Electrodeposited copper inductors for intraocular pressure telemetry," J. Micromech. Microeng., vol. 10, no. 2, pp. 124-129, Jun. 2000.

[5] J. W. McLaren, R. F. Brubaker, and J. S. FitzSimon, "Continuous measurement of intraocular pressure in rabbits by telemetry," Invest. Ophthalmol. Vis. Sci., vol. 37, no. 6, pp. 966-975, May 1996.

[6] K. C. Katuri, S. Asrani, and M. K. Ramasubramanian, "Intraocular pressure monitoring sensors," IEEE Sensors J., vol. 8, no. 1, pp. 12-19, Jan. 2008.

[7] C. R. Ethier, M. Johnson, and J. Ruberti, "Ocular biomechanics and biotransport," Аппи. Rev. Biomed. Eng., vol. 6, pp. 249-273, 2004.

[8] I. G. Pallikaris, G. D. Kymionis, H. S. Ginis, G. A. Kounis, and M. K. Tsilimbaris, "Ocular rigidity in living human eyes," Invest. Ophthalmol. Vis. Sci., vol. 46, no. 2, pp. 409-414, Feb. 2005.

[9] W. Mokwa and U. Schnakenberg, "Micro-transponder systems for medical applications," IEEE Trans. Instrum. Meas., vol. 50, no. 6, pp. 15511555, Dec. 2001

[10] W. Mokwa, "Medical implants based on microsystems," Meas. Sci. Technol., vol. 18, no. 5, pp. R47-R57, Mar. 2007.

[11] Sajeeda and T. J. Kaiser, "Passive telemetric readout system," IEEE Sens. J., vol. 6, no. 5, pp. 1340-1345, Oct. 2006.

[12] C. C. Collins, "Miniature passive pressure transensor for implanting in the eye," IEEE Trans. Biomed. Eng., vol. BME-14, no. 2, pp. 74-83, Apr. 1967.

[13] L. Rosengren, P. Rangsten, Y. Böcklund, B. Hök, B. Svedbergh, and G. Selén, "A system for passive implantable pressure sensors," Sens. Actuators A, Phys., vol. 43, no. 1-3, pp. 55-58, May 1994.

[14] O. Akar, T. Akin, and K. Najafi, "A wireless batch sealed absolute capacitive pressure sensor," Sens. Actuators A, Phys., vol. 95, no. 1, pp. 29-38, Dec. 2001.

[15] A. DeHennis and K. D. Wise, "A double-sided single-chip wireless pressure sensor," in Proc. 15th IEEE Int. Conf. MEMS, Las Vegas, NV, Jan. 20-24, 2002, pp. 252-255.

[16] A. Baldi, W. Choi, and B. Ziaie, "A self-resonant frequency-modulated micromachined passive pressure transensor," IEEE Sensors J., vol. 3, no. 6, pp. 728-733, Dec. 2003. 
[17] M. A. Fonseca, M. G. Allen, J. Kroh, and J. White, "Flexible wireless passive pressure sensors for biomedical applications," in Proc. 12th SolidState Sens., Actuators, Microsyst. Workshop, Hilton Head Island, SC, Jun. 4-8, 2006, pp. 37-42.

[18] J. Shih, J. Xie, and Y.-C. Tai, "Surface micromachined and integrated capacitive sensors for microfluidic applications," in Proc. 12th Transducers Conf., Boston, MA, Jun. 8-12, 2003, pp. 388-391.

[19] P.-J. Chen, D. C. Roger, S. Saati, M. S. Humayun, and Y.-C. Tai, "Microfabricated implantable parylene-based wireless passive intraocular pressure sensors," in Proc. 22th IEEE Int. Conf. MEMS, Sorrento, Italy, Jan. 25-29, 2009, pp. 244-247.

[20] P.-J. Chen, S. Saati, R. Varma, M. S. Humayun, and Y.-C. Tai, "Implantable flexible-coiled wireless intraocular pressure sensor," J. Microelectromech. Syst., vol. 17, no. 6, pp. 1342-1351, 2008.

[21] S. Van de Veire, P. Germonpre, C. Renier, I. Stalmans, and T. G. Zeyen, "Influences of atmospheric pressure and temperature on the intraocular pressure," Invest. Ophthalmol. Vis. Sci., vol. 49, no. 12, pp. 5392-5396, Dec. 2008.

[22] M. A. Fonseca, J. M. English, M. von Arx, and M. G. Allen, "Wireless micromachined ceramic pressure sensor for high-temperature applications," J. Microelectromech. Syst., vol. 11, no. 4, pp. 337-343, Aug. 2002.

[23] F. E. Terman, Radio Engineers' Handbook. New York: McGraw-Hill, 1943.

[24] T. H. Lee, The Design of CMOS Radio-Frequency Integrated Circuits, 2nd ed. New York: Cambridge Univ. Press, 2004.

[25] S. Timoshenko and S. Woinowsky-Krieger, Theory of Plates and Shells, 2nd ed. New York: McGraw-Hill, 1959.

[26] W. C. Young, ROARK's Formulas for Stress \& Strain, 6th ed. New York: McGraw-Hill, 1989.

[27] Certificate of Compliance, USP Biological Tests, North Amer. Sci. Assoc., Inc., Northwood, OH, 2005.

[28] L. Wolgemuth, "Assessing the performance and suitability of parylene coating," Med. Device Diagn. Ind., vol. 22, pp. 42-49, 2000.

[29] X. Q. Wang, Q. Lin, and Y.-C. Tai, "A parylene micro check valve," in Proc. 12th IEEE Int. Conf. MEMS, Orlando, FL, Jan. 17-21, 1999, pp. $177-182$.

[30] H.-S. Noh, C. Bonner, P. J. Hesketh, and G. C. Frye-Mason, "Fabrication of parylene column for micro gas chromatograph," in Proc. 7th Mechatronics Forum Int. Conf., Atlanta, GA, Sep. 2000, pp. 6-8.

[31] A. D. DeHennis, "Remotely-powered wireless monitoring systems," Ph.D. dissertation, Dept. Elect. Eng., Univ. Michigan, Ann Arbor, MI, 2004.

[32] A. V. Oppenheim and A. S. Willsky, Signals \& Systems, 2nd ed. Upper Saddle River, NJ: Prentice-Hall, 1997.

[33] P.-J. Chen, W.-C. Kuo, W. Li, Y.-J. Yang, and Y.-C. Tai, "Q-enhanced foldandbond MEMS inductors," in Proc. 3rd IEEE Int. Conf. NEMS, Sanya, China, Jan. 6-9, 2008, pp. 869-872.

[34] F. C. Flack, E. D. James, and D. M. Schlapp, "Mutual inductance of aircored coils: Effect on design of radio-frequency coupled implants," Med. Biol. Eng., vol. 9, no. 2, pp. 79-85, Mar. 1971.

[35] W. H. Ko, S. P. Liang, and C. D. F. Fung, "Design of radio-frequency powered coils for implant instruments," Med. Biol. Eng. Comput., vol. 15, no. 6, pp. 634-640, Nov. 1977.

[36] D. K. Cheng, Field and Wave Electromagnetics, 2nd ed. Reading, MA: Addison-Wesley, 1989

[37] A. Nyshadham, C. L. Sibbald, and S. S. Stuchly, "Permittivity measurements using open-ended sensors and reference liquid calibration-An uncertainty analysis," IEEE Trans. Microw. Theory Tech., vol. 40, no. 2, pp. 305-314, Feb. 1992.

[38] J.-M. Kim, D. H. Oh, J.-H. Park, J.-W. Cho, Y. Kwon, C. Cheon, and Y.-K. Kim, "Permittivity measurements up to $30 \mathrm{GHz}$ using micromachined probe," J. Micromech. Microeng., vol. 15, no. 3, pp. 543-550, Dec. 2005.

[39] J. C. Lin, P.-J. Chen, S. Saati, R. Varma, D. C. Rodger, M. S. Humayun, and Y.-C. Tai, "A minimally invasive wireless intraocular pressure sensor implant," presented at ARVO Annu. Meeting, Fort Lauderdale, FL, May 3-7, 2009.

[40] K. G. Ong, C. A. Grimes, C. L. Robbins, and R. S. Singh, "Design and application of a wireless, passive, resonant-circuit environmental monitoring sensor," Sens. Actuators A, Phys., vol. 93, no. 1, pp. 33-43, Aug. 2001.

[41] J. Coosemans, M. Catrysse, and R. Puers, "A readout circuit for an intraocular pressure sensor," Sens. Actuators A, Phys., vol. 110, no. 1-3, pp. 432-438, Feb. 2004
[42] S. F. Pichorim and P. J. Abatti, "A novel method to read remotely resonant passive sensors in biotelemetric systems," IEEE Sensors J., vol. 8, no. 1, pp. 6-11, Jan. 2008.

[43] D. Marioli, E. Sardini, M. Serpelloni, and A. Taroni, "A new measurement method for capacitance transducers in a distance compensated telemetric sensor system," Meas. Sci. Technol., vol. 16, no. 8, pp. 1593-1599, Jun. 2005.

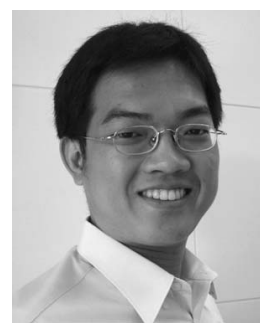

Po-Jui (PJ) Chen (S'03-M'08) was born in Taipei, Taiwan. He received the B.S. degree in mechanical engineering from National Taiwan University, Taipei, Taiwan, in 2002, and the M.S. and Ph.D. degrees in electrical engineering from California Institute of Technology (Caltech), Pasadena, in 2004 and 2008, respectively.

In 2003, he joined Dr. Yu-Chong Tai's Caltech Micromachining Laboratory as a Graduate Researcher and has been active in the area of MEMS since then $\mathrm{He}$ is currently a Research Engineer in the MEMS Technology Group, Research and Technology Center, Robert Bosch LLC, Palo Alto, CA, where he mainly works on microsensor technologies for automotive and consumer electronics applications. His research interests include new sensing concepts for physical, chemical, and biological applications; miniaturized sensors/actuators/structures; implantable biomedical microdevices; integrated microfluidic and lab-on-a-chip systems; and micro-/nanofabrication technologies. He has published more than 25 peer-reviewed journal and conference papers and is the holder of two U.S. patents and several U.S. patent applications.

Dr. Chen is a member of the American Society of Mechanical Engineers (ASME) and a full member of Sigma Xi.

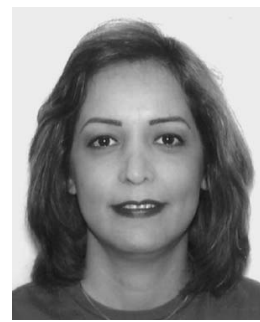

Saloomeh Saati received the M.D. degree from Iran University of Medical Sciences, Tehran, Iran, in 1999.

She finished her training by completing an ophthalmology residency at Tehran University of Medical Sciences, Tehran, Iran, in 2005. She is currently a Research Fellow of ophthalmology at the University of Southern California, Los Angeles. She is also with the Doheny Eye Institute, Los Angeles, CA. She currently works on the Artificial Retina Project and intraocular bioMEMS implants, such as drug delivery devices, intraocular pressure sensors, and shunt drainage devices.

Dr. Saati is a member of the Association for Research in Vision and Ophthalmology.

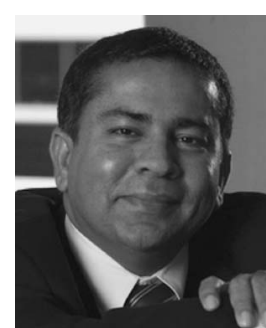

Rohit Varma received the Medical degree from the University of Delhi, Delhi, India, and the M.S. degree in public health from The Johns Hopkins University, Baltimore, MD. He completed his residency at the Wilmer Ophthalmological Institute, Johns Hopkins Hospital, and two glaucoma fellowships, one at the Wills Eye Hospital, Philadelphia, $\mathrm{PA}$, and the other at the Doheny Eye Institute, Los Angeles, CA.

He is currently a Professor of ophthalmology and preventive medicine and the Director of the Glaucoma Service, Ocular Epidemiology Center, and the Clinical Trials Unit at the Keck School of Medicine, University of Southern California, Los Angeles. As a Professor of ophthalmology, he teaches and instructs fellows and residents twice a week when he sees glaucoma patients during clinic and surgery at the Doheny Eye Institute as well as lectures on a regular basis on relevant glaucoma topics. His primary research focuses on epidemiologic studies of eye disease in children and aging populations which are both grant funded through the National Eye Institute, National Institutes of Health. He is an expert on changes in the optic nerve in glaucoma and is also studying new imaging techniques in the early diagnosis of glaucomatous optic nerve damage. More recently, he has been involved in the development of novel implantable intraocular pressure sensors and drainage devices. Due to his research, he has 110 publications in various peer-reviewed ophthalmic journals. He is the Principal Investigator of the Los Angeles Latino Eye Study, the Multi-Ethnic Pediatric Eye Diseases Study, and the Chinese American Eye Study.

Prof. Varma was the recipient of the Research to Prevent Blindness Sybil B. Harrington Award in 2003. 


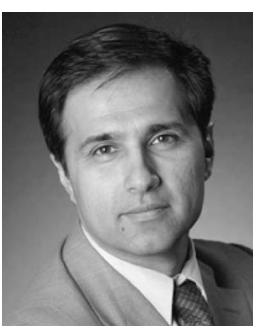

Mark S. Humayun (M'97) received the B.S. degree from Georgetown University, Washington, DC, in 1984, the M.D. degree from Duke University, Durham, NC, in 1989, and the Ph.D. degree from the University of North Carolina, Chapel Hill, in 1994. He then completed his ophthalmology residency at Duke Eye Center and fellowships in both vitreoretinal and retinovascular surgery at the Wilmer Eye Institute, Johns Hopkins Hospital, Baltimore, MD.

He stayed on as a Faculty Member at Johns Hopkins where he rose to the rank of Associate Professor before moving to the University of Southern California, Los Angeles, in 2001. He is currently a Professor of ophthalmology, biomedical engineering, and cell and neurobiology, and holds the Cornelius J. Pings Chair in biomedical sciences with the Doheny Eye Institute at the Keck School of Medicine of the University of Southern California. He is a practicing Vitreoretinal Surgeon with the Doheny Retina Institute and the Director of two national centers of excellence, and they are the National Science Foundation BioMimetic MicroElectronic Systems (BMES) Engineering Research Center and the Department of Energy Artificial Retina Project. He has authored more than 150 peerreviewed scientific papers and chapters. He is the holder of more than 75 issued or pending patent applications, and his work has spawned five companies to date, including Second Sight Medical Products Inc., a company focused on restoring sight to the blind through an implantable microelectronic artificial retina.

Dr. Humayun is a member of 13 academic organizations, including the National Academies Institute of Medicine IEEE-EMBS, the Biomedical Engineering Society, the Association for Research in Vision and Ophthalmology, the American Society of Retinal Specialists, the Retina Society, the American Ophthalmological Society, the American Academy of Ophthalmology, and Biomedical Engineering in Medicine and Biology. He has given keynote lectures in more than 20 countries. His research and clinical work has been featured prominently in more than 500 newspapers and television programs throughout the U.S. and abroad. He has been repeatedly voted as one of the Best Doctors in America and has received numerous research awards. For his outstanding contributions to biomedical engineering, he was named the Innovator of the Year in 2005 by $R \& D$ Magazine.

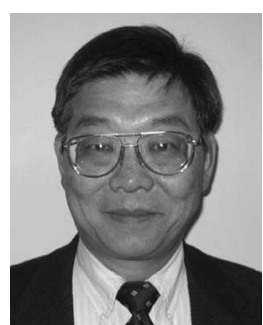

Yu-Chong Tai (M'97-SM'03-F'06) received the B.S. degree in electrical engineering from National Taiwan University in 1981, and the M.S. and Ph.D. degrees in electrical engineering from the University of California at Berkeley in 1986 and 1989, respectively.

He is currently a Professor of electrical engineering, mechanical engineering, and bioengineering at California Institute of Technology (Caltech), Pasadena. He developed the first electrically spun polysilicon micromotor at the University of California, Berkeley. At Caltech, his main research interest is MEMS for biomedical applications, including lab-on-a-chip and microimplants. He has published more than 300 technical articles in the field of MEMS.

Dr. Tai was the recipient of the IBM Fellowship, Ross Tucker Award, Best Thesis Award (at Berkeley), Presidential Young Investigator Award, Packard Award, and ALA Achievement Award. 